\title{
Planetary CHOPS
}

Ultra-Low-Cost Missions Enabled by Commercially HOsted Payload Services (CHOPS)

\section{Primary Author:}

Name: $\quad$ Adam Schilffarth

Institution: Xplore Inc.

Email: adam.schilffarth@xplore.com

Phone: 512-694-9800

\section{Co-Authors:}

S. Alan Stern, Southwest Research Institute

Dante Lauretta, University of Arizona

\section{Co-Signers}

Alan Cassell, NASA Ames

Elizabeth Frank, First Mode

Lou Friedman, Emeritus - The Planetary Society

Darren Garber, Xplore Inc.

James Garvin, NASA Goddard

Noam Izenberg, Johns Hopkins University

Georgiana Kramer, Planetary Science Institute

Robert Lillis, University of California, Berkeley

Kirby Runyon, Johns Hopkins University

Mark Sykes, Planetary Science Institute

Akbar Whizin, Southwest Research Institute

\section{ABSTRACT:}

The planetary science community's ongoing effort to obtain sufficient science flight opportunities for its investigators is limited by budgets. In this white paper we discuss a Commercially HOsted Payload Service (CHOPS), a new mission category that enables cubsesatclass payloads to fly on shared missions, on a hosted-payload basis, either in Earth orbit or beyond. By combining multiple cubesat-class payloads onto each CHOPS mission, costs are spread across multiple payload providers, allowing science to be accomplished at lower cost than dedicated missions. We propose that in addition to the traditional paths for flying instruments aboard NASA and NASA partner space agency missions, the planetary science community utilize CHOPS to provide new, frequent, ultra-low-cost options for flying scientific investigations. 


\section{Introduction}

Few domains of science are as capital-intensive as planetary science. This paper discusses a new class of missions enabled by the commercial spaceflight revolution and associated technologies: Commercially HOsted Payload Services (CHOPS). CHOPS enables cubsesat-class payloads to fly on a shared mission basis, in Earth orbit or beyond. By combining multiple payloads onto each CHOPS mission, launch and mission costs are spread across multiple payload providers, allowing science to be accomplished at lower cost than dedicated missions by individual universities or space agencies. While payload hosting has seen some adoption in Earth science and heliophysics, it is an under-appreciated mission model for planetary science.

\section{Challenges Faced by the Planetary Science Community}

As with all of the space sciences, planetary science faces two significant challenges: insufficient science opportunities to meet the needs of the community, and budgets dominated by large projects. These challenges limit scientific return, career development, and innovation. The longterm health of planetary science would benefit from ways to mitigate these challenges.

\section{Unique Strengths of Commercial Entities}

The emergence of new commercial entities in the space sector has been well documented in recent years by the media and policy analysts. Still, it is worthwhile to restate some of the unique strengths of commercial entities:

\section{- Business model efficiency}

A properly functioning commercial entity is in a constant state of cost reduction. This results in the relentless pursuit of efficiency and adaptability to match products to market need. This strong feedback loop is of great benefit to markets like planetary science which struggle to maximize the value purchased by limited budgets.

\section{- Rapid and agile innovation}

A key strength that flows from commercial motives is an incentive for rapid innovation, which in turn forces commercial entities to continually reinvent themselves and their methods to remain competitive. This can also be of great benefit to planetary science, as it also maximizes the value that can be achieved with a limited budget.

\section{CHOPS: An Ultra-Low-Cost Mission Class}

The miniaturization revolution has been a boon to space scientists looking to maximize research capabilities under competed mission cost caps, but it falls short of freeing the scientist to focus on the science. CubeSats are a low cost platform for small instruments, but they typically require the $\mathrm{PI}$ be responsible for building or procuring their own spacecraft bus. A CHOPS mission can be thought of as a commercial Partner Mission of Opportunity (PMO) where a spacecraft bus is built and flown by a commercial partner to host multiple PI payloads. 


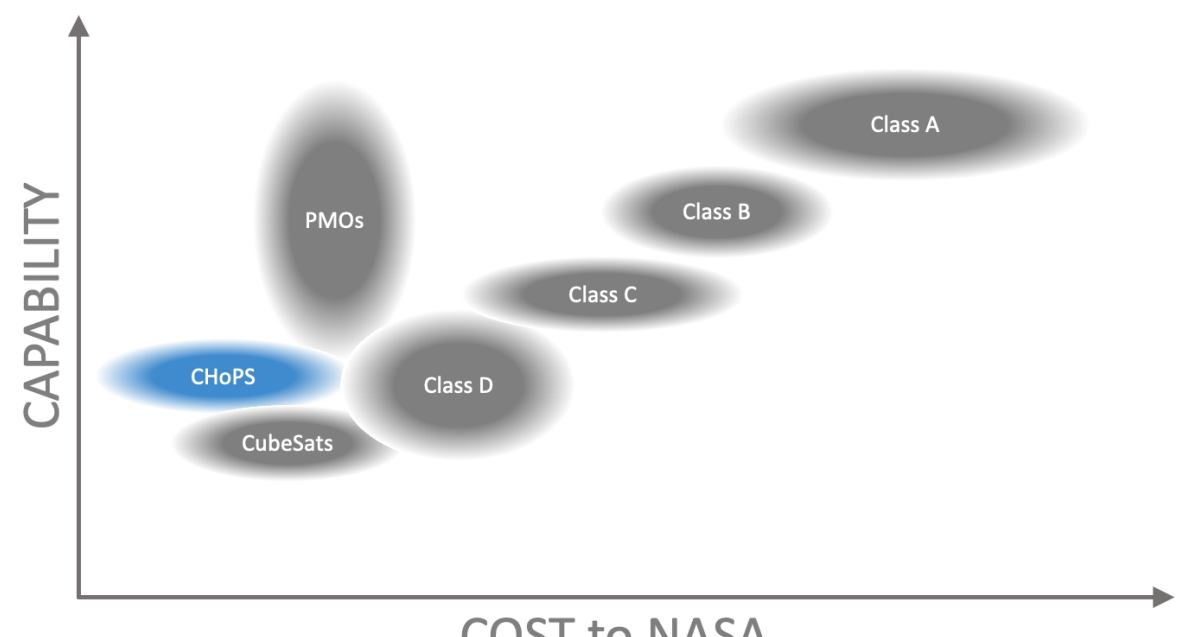

Figure 1: Qualitative illustration of how the CHOPS mission class complements other science mission classes with respect to capability versus cost. With CHOPS missions, a funding entity can get SIMPLEx-class science for less than CubeSat cost.

\section{Rapid Mission Development and Integration}

When hosting a payload on a CHOPS provider, a PI is able to focus exclusively on developing their instrument and how the instrument integrates into the CHOPS spacecraft. This way the total timeline from concept to flight is compressed compared to a stand-alone mission. Ideally, with a robust provider market, hosting an instrument will feel more like buying a plane ticket than building an airplane and learning to fly it. Pls can thus concentrate on science, not spacecraft.

\section{Cost Savings}

A CHOPS mission provides significant value alternative for small planetary missions such as SIMPLEx and cubesat missions. The cost savings in CHOPS missions come from:

\section{- Payload "Ride Sharing"}

A CHOPS mission at its core is a type of rideshare service. A single CHOPS mission spreads the mission costs across multiple payload customers. The resources available to each customer payload are contractually guaranteed in accordance with a payload hosting agreement. This model allows for a PI to purchase only as much spacecraft capability as they require.

\section{- Reduced government oversight and management}

As has been seen with hosted payloads on the Commercia Lunar Payloads Services (CLPS) program, a CHOPS mission typically has less government reporting, documentation, and auditing. The lighter oversight burden of the CHOPS model is a welcome relief to small planetary missions trying to stay within modest budgets. 


\section{- Fixed-price payload hosting}

While not a panacea, the fixed-price nature of the CHOPS model makes for a stronger proposal. When flying payloads on a CHOPS mission there is less need to carry budget margin for the spacecraft bus, communication link, or mission operations. These costs are covered by the CHOPS provider's fixed price bid and associated budget risks are carried by the CHOPS provider.

Recently, Xplore Inc. recently priced interplanetary SIMPLEx missions to inner solar system destinations as CHOPS missions for budgets starting at \$14 million for three payloads. This price includes:

- Spacecraft bus

- Payload integration

- Communication to Earth

- Spacecraft operations

Price does not include payload development, payload build, or science operations. Typically, a CHOPS cost estimate would include launch, but in this case, per the SIMPLEx guidelines, launch was excluded.

\section{CHOPS compared to CubeSats}

Taking a cubesat-class instrument off of the cubesat and hosting it on a larger spacecraft opens up a number of capability enhancements at a lower cost than flying a stand-alone cubesat mission:

\section{- Power}

The power available on smallsats (and larger) is a significant capability improvement over even the largest CubeSat bus. Having more power available frees up additional scarce resources on the spacecraft such as pointing and communication time.

\section{- Communications}

CHOPS spacecraft can provide larger communication rates than cubesats. This not only means more returned data, but also less time needed for communications pointing, and more time available for instrument pointing.

- Mission complexity and duration

CHOPS spacecraft can have more robust propulsion and attitude control than cubesats, enabling more complex mission design, better pointing stability, and longer mission duration. 


\section{- High Performance Computing}

An emerging trend in science mission design is the incorporation of high-performance computers onboard spacecraft to create higher order data products in an effort to reduce the quantity of downlinked data. Although this is a promising technological innovation, it is seen by many in the community as too "risky" to incorporate in their proposals. CHOPS class missions are an excellent opportunity to mature onboard data reduction practices at ultra-low-cost.

Table 1: Listing of CHOPS providers by hosting location.

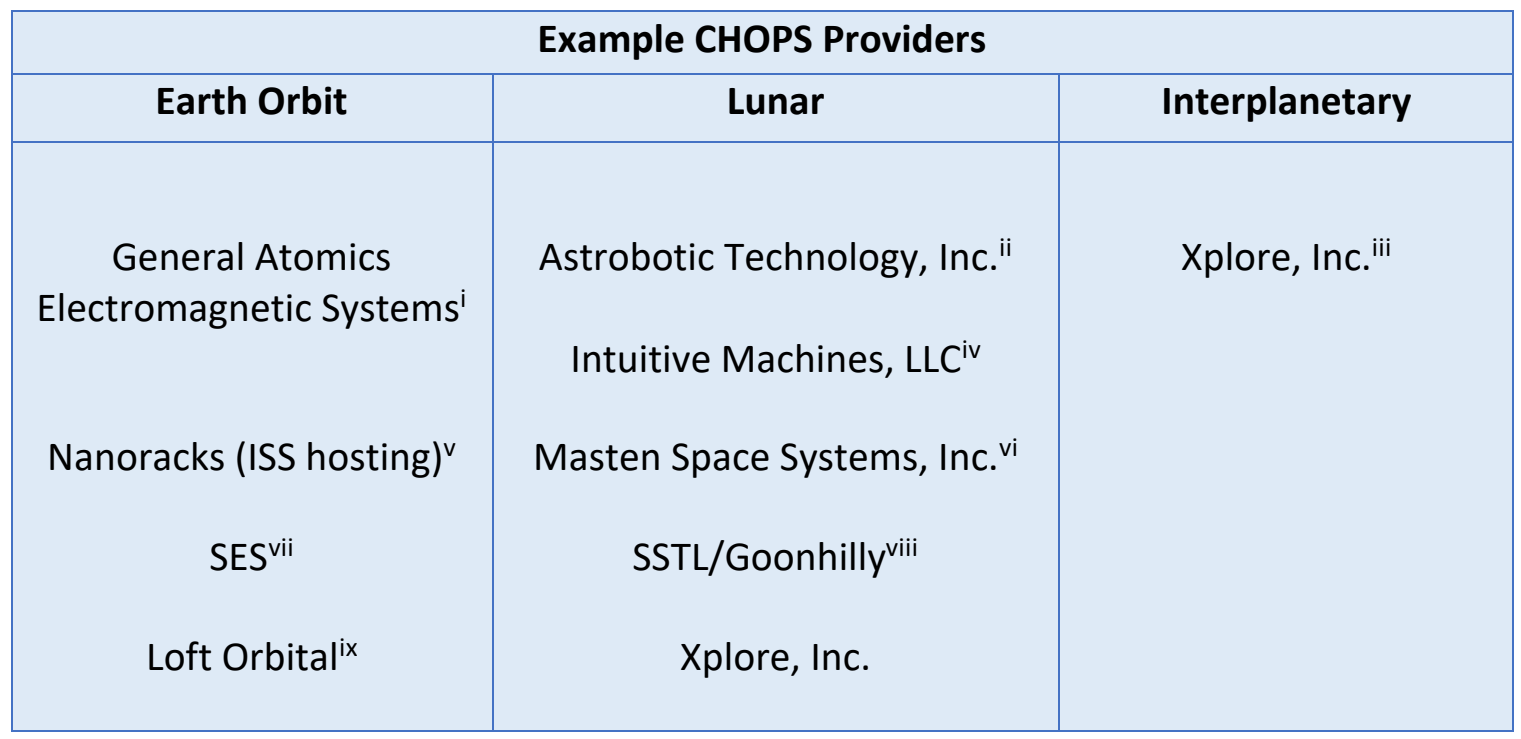

\section{Benefits to Planetary Science Community}

CHOPS class missions are particularly compelling in the context of the Planetary Science community's needs. By giving the community an ultra-low-cost mission class that does not require spacecraft development by PIs or their institutions, PIs of all career stages and institutions can fly instruments quicker, more often, and at lower cost. New CHOPS providers entering the market are opening up destinations throughout the inner solar system to this new ultra-low-cost mission class.

\section{Expansion of Participation}

By lowering the financial barrier to entry, CHOPS enables participation in planetary science by groups who previously could not or do not wish to undertake cubesat construction and operations. Groups looking to break into flying planetary science missions can start with flying a single hosted payload for a modest price to grow their program over time. 


\section{Early Career Development}

CHOPS are particularly attractive as a mechanism for developing early career scientists into PIs. A CHOPS mission puts little agency capital at risk and does not displace other larger missions from launch opportunities. A successful CHOPS mission is a natural stepping-stone toward being the PI for a CubeSat mission which then opens doors to Class D missions. A typical CHOPS mission is a fraction of the SIMPLEx cost cap and programmatically resembles a traditional Partner Mission of Opportunity (PMO).

\section{"For a PI, demonstrated leadership is a must." \\ - Dr. Thomas Zurbuchen, NASA National Colloquium, June $5^{\text {th }}, 2019$}

\section{Technology Maturation}

In addition to giving PIs "flight heritage," CHOPS missions are also an opportunity to give flight heritage to innovative technologies that are too immature to fly on more expensive, standalone missions. A CHOPS provider's tolerance for risk scales with revenue potential, so a highrisk technology simply means that the hosting fee scales proportionally. Traditional PMO entities are disinclined to allow a high-risk technology on their large mission.

\section{Ultra-Low-Cost}

CHOPS allows for SIMPLEx-class science at a cost below CubeSat missions. Hosting fees for CHOPS missions are on the order of $\$ 1 \mathrm{M}-2 \mathrm{M}$ per kilogram which means that the typical SIMPLEx-class instrument could be hosted for less than the cost of a deep space cubesat mission. ${ }^{x}$ Often a smallsat mission's budget is no more than $20 \%$ science, which means that a funding entity is paying $5 x$ what the science really costs. By hosting instruments on CHOPS missions, a funding entity can see the non-science overhead drop to being on par with the cost of the science itself.

A CHOPS hosted mission potentially allows for a small Discovery Class Mission to be done for the cost of a SIMPLEX Mission. 


\section{CHOPS SWOT Chart}

A SWOT analysis is helpful in framing where CHOPS missions are best positioned.

Table 2: Notional SWOT chart for CHOPS.

\begin{tabular}{|l|l|}
\hline \multicolumn{1}{|c|}{ Strengths } & \multicolumn{1}{c|}{ Weaknesses } \\
\hline $\begin{array}{l}\text { Lowers mission costs. } \\
\text { Allows PIs to purchase only as much } \\
\text { capability as they need. }\end{array}$ & $\begin{array}{l}\text { A mission may not fly if the mission } \\
\text { provider does not contract enough } \\
\text { payloads. }\end{array}$ \\
$\begin{array}{l}\text { Competition compels CHOPS } \\
\text { providers to seek efficiency and } \\
\text { improve performance. }\end{array}$ & $\begin{array}{l}\text { NASA and other government } \\
\text { customers are initially needed to } \\
\text { create a market. }\end{array}$ \\
$\begin{array}{l}\text { Removes the need for the PI to build } \\
\text { their own spacecraft as they must } \\
\text { with cubesats. }\end{array}$ & $\begin{array}{l}\text { Opportunities } \\
\text { Opportunities increased for new } \\
\text { entrants into planetary science (e.g., } \\
\text { smaller nations). }\end{array}$ \\
\hline
\end{tabular}

\section{Recommendation}

We respectfully recommend that the Decadal Survey endorse CHOPS as an ultra-low-cost method for planetary science and planetary defense missions, thereby increasing the number and diversity of mission types that NASA can engage in planetary exploration.

\footnotetext{
i https://eoportal.org/web/eoportal/satellite-missions/content/-/article/otb-1

ii https://www.astrobotic.com/

iii https://www.xplore.com/xpeditions/custom.html

iv https://www.intuitivemachines.com/lunarlander

$\checkmark$ https://nanoracks.com/products/external-platform/

vi https://www.masten.aero/moon

vii https://gold.cs.ucf.edu/gold-installed-on-commercial-communications-satellite/

viii https://www.goonhilly.org/lunar

ix https://www.loftorbital.com/\#about

${ }^{x}$ https://phys.org/news/2018-11-big-tiny-satellites-trailing-mars.html
} 